\title{
Myocardial hypertrophy in the absence of external stimuli is induced by angiogenesis in mice
}

\author{
Daniela Tirziu, ${ }^{1}$ Emmanuel Chorianopoulos, ${ }^{2}$ Karen L. Moodie, ${ }^{1}$ Robert T. Palac, ${ }^{1}$ \\ Zhen W. Zhuang, ${ }^{1}$ Marc Tjwa, ${ }^{2}$ Carmen Roncal, ${ }^{2}$ Ulf Eriksson, ${ }^{3}$ Qiangwei Fu, ${ }^{1}$ \\ Arye Elfenbein, ${ }^{1}$ Amy E. Hall, ${ }^{1}$ Peter Carmeliet, ${ }^{2}$ Lieve Moons, ${ }^{2}$ and Michael Simons ${ }^{1}$
}

\begin{abstract}
${ }^{1}$ Angiogenesis Research Center, Section of Cardiology, Department of Medicine, and Department of Pharmacology and Toxicology, Dartmouth Medical School, Hanover, New Hampshire, USA. ${ }^{2}$ Department for Transgene Technology and Gene Therapy and Center for Transgene Technology and Gene Therapy, Katholieke Universiteit Leuven, Leuven, Belgium. ${ }^{3}$ Ludwig Institute for Cancer Research, Stockholm Branch, Stockholm, Sweden.
\end{abstract}

\begin{abstract}
Although studies have suggested a role for angiogenesis in determining heart size during conditions demanding enhanced cardiac performance, the role of EC mass in determining the normal organ size is poorly understood. To explore the relationship between cardiac vasculature and normal heart size, we generated a transgenic mouse with a regulatable expression of the secreted angiogenic growth factor PR39 in cardiomyocytes. A significant change in adult mouse EC mass was apparent by 3 weeks following PR39 induction. Heart weight; cardiomyocyte size; vascular density normalization; upregulation of hypertrophy markers including atrial natriuretic factor, $\beta$-MHC, and GATA4; and activation of the Akt and MAP kinase pathways were observed at 6 weeks post-induction. Treatment of PR39-induced mice with the eNOS inhibitor L-NAME in the last 3 weeks of a 6-week stimulation period resulted in a significant suppression of heart growth and a reduction in hypertrophic marker expression. Injection of PR39 or another angiogenic growth factor, VEGF-B, into murine hearts during myocardial infarction led to induction of myocardial hypertrophy and restoration of myocardial function. Thus stimulation of vascular growth in normal adult mouse hearts leads to an increase in cardiac mass.
\end{abstract}

\section{Introduction}

Factors determining organ size in an adult organism are poorly understood. Several lines of evidence suggest that angiogenesis is required for organ enlargement for tissue repair or in response to a physiological stimulus (1-3). However, little is known about the effect of vascular mass on the normal organ size.

Myocardial size and function in adult hearts remain stable in the absence of external stimuli that demand enhanced cardiac performance, such as increased afterload or a partial loss of tissue due to infarction or other injury. When such circumstances arise, the heart meets increased hemodynamic demand by undergoing chamber enlargement and myocyte hypertrophy $(4,5)$. The adaptation fails at high loads, secondary to the development of tissue ischemia due to a mismatch between myocardial oxygen supply and demand, as such myocardial enlargement is typically not accompanied by a corresponding adequate increase in the arterial blood supply $(6,7)$. Enhancement of angiogenesis in the failing heart is reported to improve myocardial function (8).

In order to address the question of whether an increase in the cardiac vasculature in the absence of other stimuli can drive myocardial hypertrophy, we used an inducible mouse transgenic model that allows activation and deactivation of expression of the secreted angiogenic factor PR39 in cardiomyocytes. Previous studies in PR39 transgenic mice demonstrated marked

Nonstandard abbreviations used: Ad-hVEGF- ${ }_{167}$, adenovirus encoding human VEGF-B 167 ; Ad-PR39, adenovirus encoding PR39; ANF, atrial natriuretic factor; LAD left anterior descending; MI, myocardial infarction; RGS, regulators of G-protein signaling; TRE, tetracycline-responsive element; tTA, tetracycline transactivator. Conflict of interest: The authors have declared that no conflict of interest exists. Citation for this article: J. Clin. Invest. 117:3188-3197 (2007). doi:10.1172/JCI32024. induction of angiogenesis by this growth factor (9). To set up a regulatable myocardial PR39 expression, PR39 gene was placed under control of a tetracycline-responsive element (TRE) promoter sensitive to tetracycline transactivator ( $\mathrm{tTA}$ ) regulated by $\alpha$-MHC promoter.

Using this model, we showed that an increase in the endothelial cell mass in the normal heart in the absence of hemodynamic load leads, several weeks later, to induction of myocardial hypertrophy and that this process stops when capillary density of the myocardial tissue returns to baseline levels. Stimulation of angiogenesis with PR39 or VEGF-B at the time of myocardial infarction (MI) similarly induced angiogenesis and hypertrophy, improving myocardial function. What we believe to be a novel mechanism of endothelium-driven myocardial hypertrophy may be a fundamental factor in regulating heart size and may provide new avenues of research for therapeutic strategies designed to improve cardiac function.

\section{Results}

Inducible PR39 expression in vitro and in vivo. To verify effectiveness of the "tet-off" promoter system in driving PR39 expression, the TRE-PR39/LacZ bidirectional construct was expressed in $\mathrm{MEF} / 3 \mathrm{~T} 3$ cells stably expressing the LTR-tTA transgene. Withdrawal of doxycycline led to a marked increase in $\beta$-galactosidase expression that was comparable to that achieved by CMV promoter-driven expression and was completely suppressible by reintroduction of doxycycline (Figure 1A). The TRE-PR39/ LacZ bidirectional construct was then transgenically expressed in C57BL/6 mice. PCR analysis of PR39/LacZ expression in the hearts of 7-week-old mice confirmed that PR39 mRNA expression was activated by doxycycline withdrawal and was fully deac- 
A

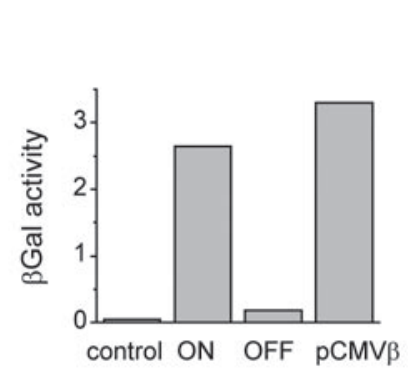

B

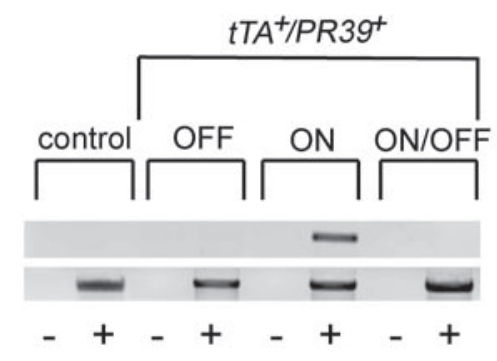

C
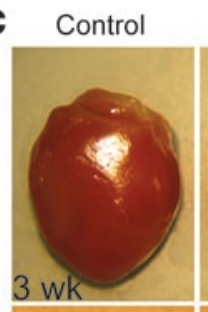

GAPDH


H

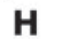

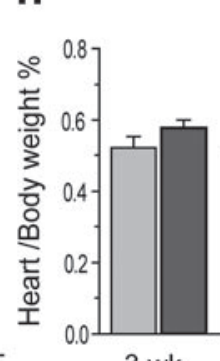

3 wk
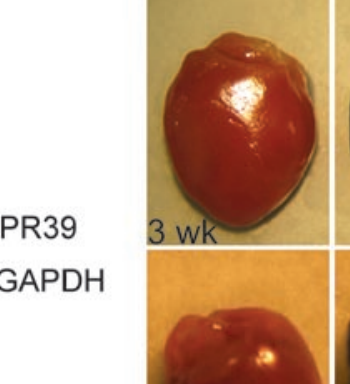

6 wk

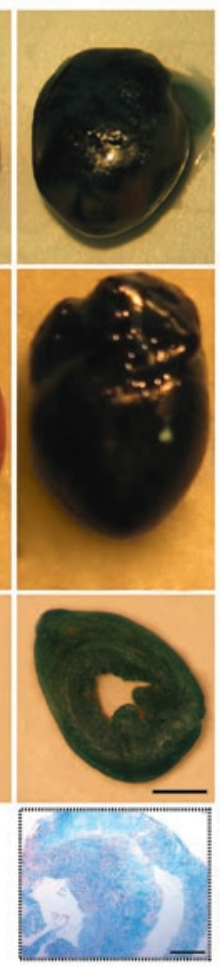

tTATPR39+

Figure 1

Inducible transgenic PR39/LacZ expression. (A) $\beta$-Galactosidase activity was induced in Tet-off-inducible MEF/3T3 cells (control) transfected with TRE-PR39/LacZ vector in the absence of doxycycline (ON). In the presence of doxycycline (OFF), $\beta$-galactosidase expression was completely abolished. A similar extent of $\beta$-galactosidase activity was achieved with the non-inducible reporter vector pCMV $\beta$. (B) RT-PCR analysis of PR39 transcript in mouse hearts. Note expression only in induced $t T A^{+} / P R 39^{+}$mice $(\mathrm{ON})$ and resuppression of expression by doxycycline (ON/OFF). (C) Hearts of controls and $t T A^{+} / P R 39^{+}$mice after 3 weeks (top row) and 6 weeks of PR39 induction (middle and bottom rows). Note an increase in the heart size and LV chamber after 6 weeks ON and the extensive expression of the transgene as demonstrated by X-Gal staining (right column). Lower right panel: X-gal-stained LV cross-section. Scale bar: $2 \mathrm{~mm} ; 1 \mathrm{~mm}$ (inset). (D and E) Total cardiac endothelial cell mass determined by in vivo anti-PECAM Ab labeling (D) or capillary counting (E). Note a significant increase in endothelial cell mass at week 3 that is maintained at week 6 ( $n=5$ mice/group). (F) Myocardial endothelial cell mass determined by in vivo anti-PECAM Ab labeling expressed per gram of heart tissue. Note normalization of the endothelial cell mass/g tissue at 6 weeks. (G) Heart weight measurements after 3 and 6 weeks of PR39 induction and 6 weeks ON followed by 3 weeks of suppression (6ON/3OFF), compared with controls. (H) Heart weight/body weight ratio determined after 3 weeks, 6 weeks ON, and 6ON/3OFF. ${ }^{*} P<0.05,{ }^{*} P<0.01$. 3 wk, $n=10$ mice/group; 6 wk, $n=16$ mice/group; $6 \mathrm{ON} / 3 \mathrm{OFF}, n=10 \mathrm{mice} / \mathrm{group}$.

tivated by its reintroduction (Figure $1 \mathrm{~B}$ ), and X-gal staining of the entire heart demonstrated the homogenous nature of transgene expression (Figure 1C).

Angiogenesis induction in the adult mouse heart. To explore the effect of angiogenic stimulation in the adult heart on its vasculature, size, and function, PR39 expression was activated in the hearts of 5-week-old mice. In agreement with previous studies (9), this was associated with an increase in HIF-1 $\alpha$ and iNOS expression (Supplemental Figure 1; supplemental material available online with this article; doi:10.1172/JCI32024DS1). Three weeks later, there was a significant increase in the cardiac endothelial cell mass as determined by in vivo perfusion with an $\mathrm{I}^{125}$-labeled anti-PECAM antibody (Figure 1D), with an increase in the capillary count (Figure 1E) and amount of vasculature per gram of tissue (Figure 1F).
At the same time, there was no increase in the heart weight or the heart weight/body weight ratio (Figure 1, C, G, and H).

After 6 weeks of continued stimulation of PR39 expression, the increase in the endothelial cell mass was unchanged (Figure 1, D and $\mathrm{E}$ ) but there was a large increase in the heart size and weight and the heart weight/body weight ratio (Figure 1, C, G, and H). Interestingly, quantification of endothelial cell mass showed that the vascular density per gram of myocardial tissue had returned to values seen in control mice (Figure 1F). The increases in the heart weight and heart weight/body weight ratio were maintained 3 weeks after discontinuation of PR39 stimulation (Figure 1, G and $\mathrm{H}$ ), suggesting that size changes were permanent.

Induction of myocardial bypertrophy. Morphologic examinations of the heart after 6 weeks of PR39 stimulation demonstrated 
A

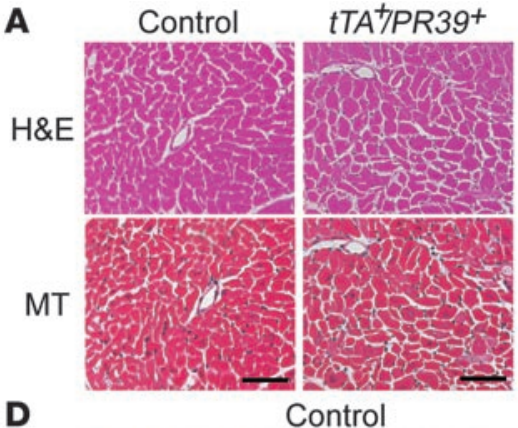

D


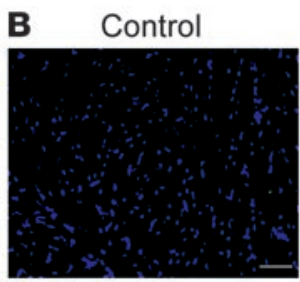

tTAt/PR39+

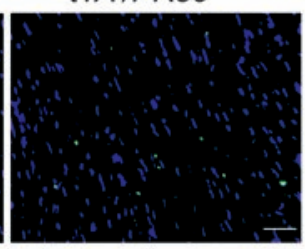

DAPI

TUNEL

tTA ${ }^{+} / P R 39^{+}$
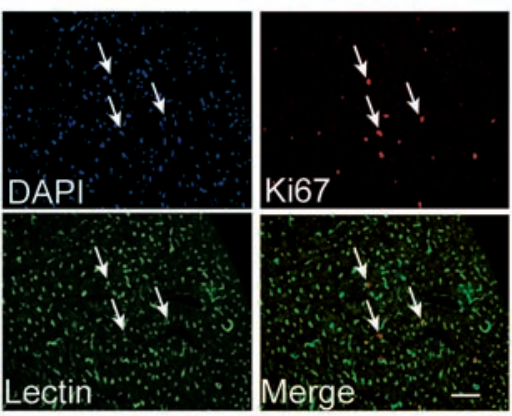

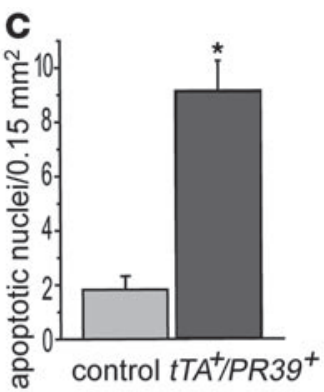

E

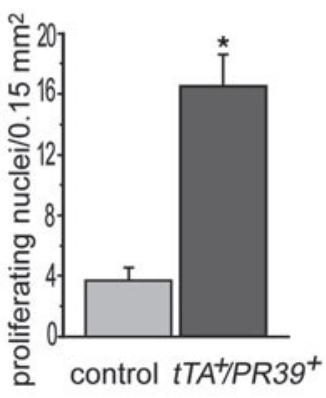

\section{Figure 2}

Histological analysis of $t T A^{+} / P R 39^{+}$hearts after 6-weeks induction. (A) Interstitial fibrosis as assessed by Masson's trichrome staining (MT). Note the absence of fibrosis in $t T A^{+} / P R 39^{+}$mice induced for 6 weeks. (B and C) Apoptosis assessed by double DAPI and TUNEL staining (B) and its quantification (C). (D) Cell proliferation assessed with anti-Ki67 Ab and DAPI and lectin staining. Note association of Ki67 antigen expression with endothelial cells (arrows). (E) Quantification of proliferation. Note an increase of cell proliferation in $t T A^{+} / P R 39^{+}$mice compared with control mice. ${ }^{*} P<0.001$ ( $n=5$ mice/group). Scale bars: $50 \mu \mathrm{m}$.

increased ventricular wall thickness and an increase in the LV chamber size (Figure 1C). There was no noticeable increase in the extent of fibrosis (Figure 2A), although there was a small but significant increase in apoptosis (Figure 2, B and C). Immunocytochemical staining with anti-Ki67 antibody demonstrated increased proliferation of endothelial cells between myocyte bundles, but no proliferation of cardiomyocytes was noted in multiple sections (Figure 2, D and E).

Examination of enzymatically dispersed cardiomyocytes showed a marked increase in their cross-sectional area proportionate to the overall increase in heart size (45\% heart enlargement vs. $30 \%$ myocyte area increase) (Figure 3, A and B). Taken together, these findings suggest that myocyte enlargement was the principal cause of the increased heart size.

To gain further insight into the nature of the hypertrophy process, we studied expression of myocyte hypertrophy markers. As early as 3 weeks, there was a mild increase in $\beta$-MHC expression that was quite prominent by 6 weeks. A marked increase in atrial natriuretic factor (ANF) and skeletal $\alpha$-actin gene expression was also noted at 6 but not 3 weeks (Figure 3C). Similarly, by 6 weeks there was an increase in phosphorylated Akt (1.6-fold), phosphorylated and total Erk1/2 (2-fold), and GATA4 (2-fold) expression in PR39-stimulated hearts (Figure 3D). Three weeks following discontinuation of PR39 stimulation, the increased level of expression of hypertrophic markers and activation of Erk1/2 and GATA4 were still maintained (Figure 3, C and D).

These findings are consistent with the notion that an increase in the endothelial cell mass preceded an increase in the cardiac size that in turn was largely driven by myocyte hypertrophy. To exclude the possibility that PR39 itself, and not an increase in the endothelial cell mass, was responsible for myocardial hypertrophy, neonatal rat myocytes were transduced in vitro with adenovirus encoding PR39 (Ad-PR39) or treated with PR39 or angiotensin-II peptides. Three days later, there was a significant increase in the cell area of angiotensin-II- but not PR39-treated cardiomyocytes (Figure 4, A and D), and there was no activation of the hypertrophy program by PR39 treatment (Figure 4, B and D). Furthermore, while angiotensin-II treatment stimulated protein synthesis as assessed by ${ }^{3} \mathrm{H}$-leucine incorporation, PR39 treatment did not (Figure 4C). Taken together, these studies suggest that direct PR39 stimulation is not per se hypertrophic.

Cardiac performance. To determine whether this endothelial-driven myocardial hypertrophy affected cardiac performance, we performed echocardiographic and pressure-volume loop analyses of myocardial function. In agreement with morphologic observations, after 6 weeks of PR39 induction, there was a significant increase in the echo-determined LV posterior wall thickness (Figure $5 \mathrm{~A}$ ). At the same time, there was also a significant $\mathrm{LV}$ chamber size increase as demonstrated by an increase in LV end-diastolic and -systolic dimensions (Figure 5, $\mathrm{B}$ and $\mathrm{C}$ ). Consistent with this picture of myocardial dilatation, there was a reduction in $L V$ anterior and posterior wall fractional shortening (Figure 5D). Pressure-volume loop analysis of cardiac performance after 6 weeks of PR39 induction showed a significant decrease in both systolic (reduction in cardiac output, stroke volume, and $+\mathrm{d} P / \mathrm{d} t$ ) and diastolic (increased $\tau$ and decreased $-\mathrm{d} P / \mathrm{d} t$ ) function (Table 1). Interestingly, 3 weeks after cessation of PR39 induction, despite morphologic evidence of continued myocardial enlargement, both systolic and diastolic performance were considerably improved compared with 6 weeks of continued PR39 stimulation and even enhanced compared with control animals (Table 1). 
A

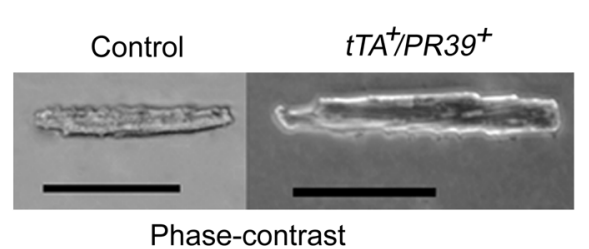

C

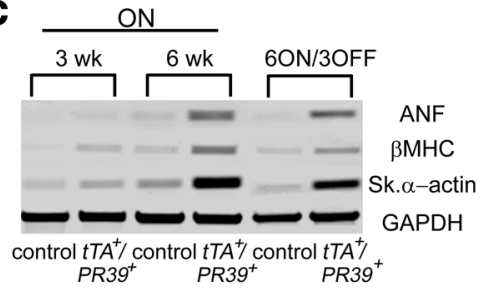

B

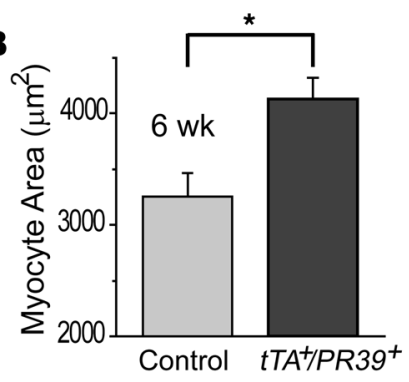

D

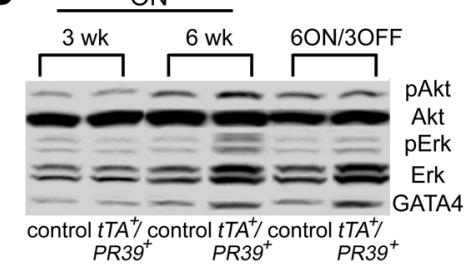

\section{Figure 3}

Myocardial hypertrophy in $t T A^{+} / P R 39^{+}$mice. (A) Isolated cardiomyocytes from $t T A^{+} / P R 39+$ mice after 6 weeks induction compared with control. Scale bar: $100 \mu \mathrm{m}$. (B) Quantification of cross-sectional area of isolated cardiomyocytes. 50 cells/ group; ${ }^{*} P<0.01$. (C) RT-PCR analysis of hypertrophic markers (ANF, $\beta$-MHC, skeletal $\alpha$-actin) after PR39 induction for 3 weeks, 6 weeks, and 6ON/3OFF. GAPDH expression was used as a control. Note the high level of expression of hypertrophic markers for 6ON/3OFF compared with control (D). Western blot of Akt activation (phospho-Akt-Ser473), Erk1/2 (phosphop44/42 MAPK Tyr202/Thr204), and transcription factor GATA4 after PR39 induction for 3 weeks, 6 weeks, and 6ON/3OFF.
eNOS as a bypertrophy modulator. NO may play a role in mediation of hypertrophy because of its effect on degradation of regulators of G-protein signaling (RGS) proteins (10). Increased production of cGMP in the hearts of mice expressing both $\alpha$-MHC-tTA and TRE-PR39 transgenes, resulting in tTA-driven myocyte-specific PR39 expression (tTA/PR39 mice) (Figure 6A), suggested the possibility that increased NO production due to increased endothelial cell mass may mediate the observed hypertrophy. In order to evaluate this potential effect of endothelial NO production, PR39-induced and control mice were treated with eNOS inhibitor L-NAME in the last 3 weeks of a 6 -week stimulation period. L-NAME treatment slightly increased systolic pressure in control mice compared with untreated controls ( $91.13 \pm 5.4$ vs. $84.25 \pm 2.8 \mathrm{mmHg}, n=5 ; P=\mathrm{NS})$ and considerably improved it in PR39-induced mice relative to untreated controls $\left(82.87 \pm 1.8\right.$ vs. $66.63 \pm 14.1$ untreated $\mathrm{tTA}^{+} / \mathrm{PR} 39^{+}$ mice, $n=5 ; P<0.01$ ), likely due to an improvement in cardiac performance (see below). L-NAME treatment resulted in a 50\% reduction of the increase in heart size caused by PR39 expression (Figure 6B) and a reduction in hypertrophic marker gene expression (ANF, $\beta$-MHC, and skeletal $\alpha$-actin) (Figure 6C). At the same time, there was no reduction in the capillary density (Figure 6D).

Interestingly, the reduction of caveolin-1 (2.5-fold; Figure 6E) and caveolin-3 (3.5-fold; Figure 6E) expression in PR39-induced mice was restored following L-NAME treatment to the levels seen in controls. This suggests a possible correlation between caveolin levels and eNOS activity and/or NO release.

Consistent with a reduction in heart size, PR39-induced mice treated with L-NAME demonstrated no enlargement of LV posterior wall thickness (Figure $6 \mathrm{~F}$ ) and a significantly reduced increase in the LV end-systolic dimensions (Figure 6G). Moreover, L-NAME treatment improved systolic and diastolic performance in PR39induced mice (Table 1).

Angiogenesis-driven hypertrophy in the setting of MI. To clarify the effect of angiogenesis-driven hypertrophy on myocardial function in pathological settings, the left anterior descending (LAD) coronary artery in $t \mathrm{TA}^{+} / \mathrm{PR} 39^{+}$mice was ligated and PR39 expression induced at the same time. In agreement with our prior results $(9,11)$, infarct size was reduced when PR39 was expressed in cardiomyocytes (Figure 7A), while ejection fraction was increased (Figure 7B). Histological examination of the free wall myocardium demonstrated a significant increase in the myocyte area 4 weeks later (Figure 7, C and D).
To verify that the angiogenesis-hypertrophy effect is not PR39 specific, C57BL/6 mice were subjected to an intravenous injection of adenovirus encoding human VEGF-B ${ }_{167}\left(\right.$ Ad-hVEGF-B ${ }_{167}$ ) a predominantly cardiac-specific angiogenic growth factor (12). Intravenous injection of Ad-hVEGF-B ${ }_{167}$ at the time of LAD ligation resulted in prolonged systemic expression and transient elevation of circulating VEGF-B ${ }_{167}$ levels (VEGF-B plasma levels, in $\mathrm{ng} / \mathrm{ml}$ : $5.7 \pm 0.5,5.5 \pm 0.3,4.8 \pm 0.5$, and $2.4 \pm 0.6$ at $3,7,14$, and 21 days after MI, $n=10 ; P<0.05$; compared with undetectable levels on each day, following Ad-RR5 injection [control]). Unlike PR39, VEGF-B administration did not reduce the infarct size 28 days after MI, and it was the same in both Ad-hVEGF- $\mathrm{B}_{167}$ and Ad-RR5 groups (Figure 7E). However, similar to PR39 mice, Ad-hVEGF-B 167 $^{- \text {treat- }}$ ed mice demonstrated a significant increase in capillary density in the remote myocardial area (Figure 7F) and an increase in the capillary/cardiomyocyte ratio (Figure 7G). Histological examination of the LV free wall remote from the infarct area demonstrated an increase in cardiomyocyte area, consistent with a hypertrophic effect (Figure 7, H and I). Overall, VEGF-B treatment was associated with improved LV function (Figure $7 \mathrm{~J}$ ).

\section{Discussion}

These results demonstrate that an increase in the organ's endothelial cell mass can drive an increase in its size in the absence of hemodynamic or any other known hypertrophy stimulus. Remarkably, the increase in mass stopped when the vessel density per gram of tissue returned to control levels, suggesting that vessel density might be the crucial parameter regulating heart size. Finally, the increase in the heart size was largely mediated by an increase in myocyte diameter and not by myocyte proliferation.

PR39 is a secreted peptide that rapidly accumulates in the extracellular matrix and is thought to act via HIF- $1 \alpha$-mediated increase in VEGF and stimulation of fibroblast growth factor signaling (9). The inducible mode of its expression employed in this study allowed us to evaluate a temporal relationship between the onset of angiogenesis and the onset of hypertrophy in the mature heart. The observed increase in the vascular mass that preceded the onset of hypertrophy is in agreement with an earlier study that demonstrated that thyroxin treatment-induced myocardial hypertrophy is preceded by an increase in the capillary and arteriolar length-volume relationship (13). The observed maintenance of the increased vascular 

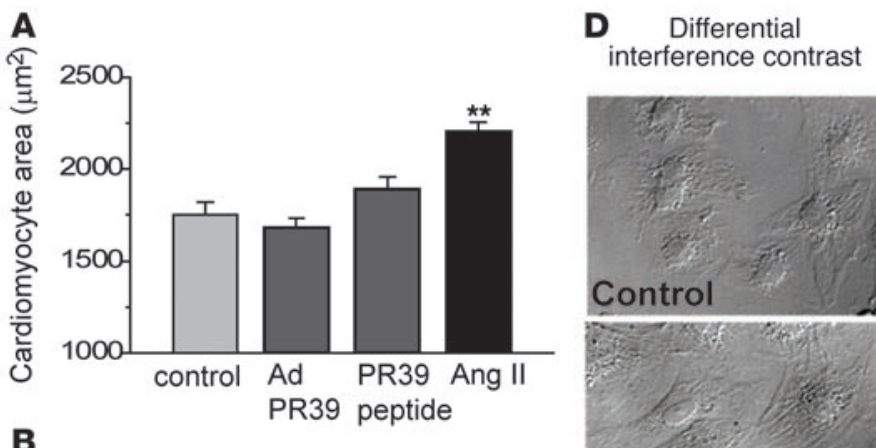

\section{Sk. $\alpha$ Actinin sarcomeric}


\section{Figure 4}

In vitro effects of PR39 administration. (A) Surface area of rat neonatal cardiomyocytes transduced with Ad-PR39 (100 MOI) or treated with PR39 $(1 \mu \mathrm{M})$ or Ang II (1 $\mu \mathrm{M})$ peptides for 72 hours $(n=150$ cells each). (B) RT-PCR analysis of hypertrophic markers ANF, $\beta$-MHC, and skeletal $\alpha$-actin after 72 hours of exposure to PR39 (adenoviral vector or peptide) or Ang II. (C) Stimulation of protein synthesis assessed by ${ }^{3 \mathrm{H}}$-leucine incorporation ( $n=3$ independent experiments). (D) Micrographs of rat neonatal cardiomyocytes transduced with Ad-PR39 (100 MOI) or treated with PR39 peptide $(1 \mu \mathrm{M})$ or Ang II $(1 \mu \mathrm{M})$ for 72 hours, immunostained with $\alpha$-actinin sarcomeric Ab. ${ }^{* *} P<0.01$. Scale bars: $50 \mu$ m.

mass after 6 weeks of stimulation followed by 3 weeks of withdrawal is consistent with the observation of Dor et al. that after a sufficient amount of continuous growth factor stimulation ( $\sim 30$ days), newly formed myocardial vasculature becomes permanent (14).

The molecular signature of the observed hypertrophic response appears very similar to that of pressure-induced hypertrophy rather than a physiologic hypertrophic response. However, the effect of the observed changes on cardiac function was complex. Continued stimulation with PR39 for 6 weeks resulted in decreased systolic and diastolic performance. Cessation of PR39 expression led to a rapid recovery of cardiac function that improved to supraphysiologic levels. It is interesting to compare these observations with cardiac function changes seen in the case of continuous transgenic Akt-1 expression, in which short-term stimulation results in supraphysiologic performance, while long-term stimulation leads to pronounced heart failure (7).

One potential mechanism that might explain both the hypertrophic response to increased endothelial cell mass and changes in cardiac function is the endothelium-dependent production of NO. Associated with NO production, alterations in caveolin-1 abundance change vessels' ability to relax. Accordingly, increase in caveolin-1 expression has been associated with hypertension and its absence with vasodilatation due to eNOS hyperactivation $(15,16)$. Moreover, the loss of caveolin-3 expression activates p42/44 MAPK cascade and induces cardiac hypertrophy (17). Recently it was suggested that NO controls the levels of RGS (10). In particular, RGS4 is thought to be involved in hypertrophic response as a negative modulator of Gq-protein signaling. Low NO levels favor RGS4 stabilization by preventing ubiquitination and proteasome-mediated degradation and thereby reducing $\mathrm{Gq}$ signaling. The increased endothelial cell mass would lead to increased NO production that would in turn lead to increased RGS4 degradation and derepression of the hypertrophic program. Our results are consistent with this hypothesis, as L-NAME treatment in a dose known to inhibit eNOS signaling reduced myocardial hypertrophy. Most interestingly, despite the fact that L-NAME treatment reduced the extent of myocardial hypertrophy, it had no effect on the increase in endothelial cell mass and capillary density. This "uncoupling" of the endothelial mass from the hypertrophic effect by L-NAME further points to NO as the molecule responsible for angiogenesis-induced hypertrophy. At the same time, the excessive production of NO at the peak of PR39 stimulation could explain the observed decrease in cardiac performance, given the ability of $\mathrm{NO}$ to reduce myocardial function and the ability of L-NAME to restore it $(16,18,19)$. 
A
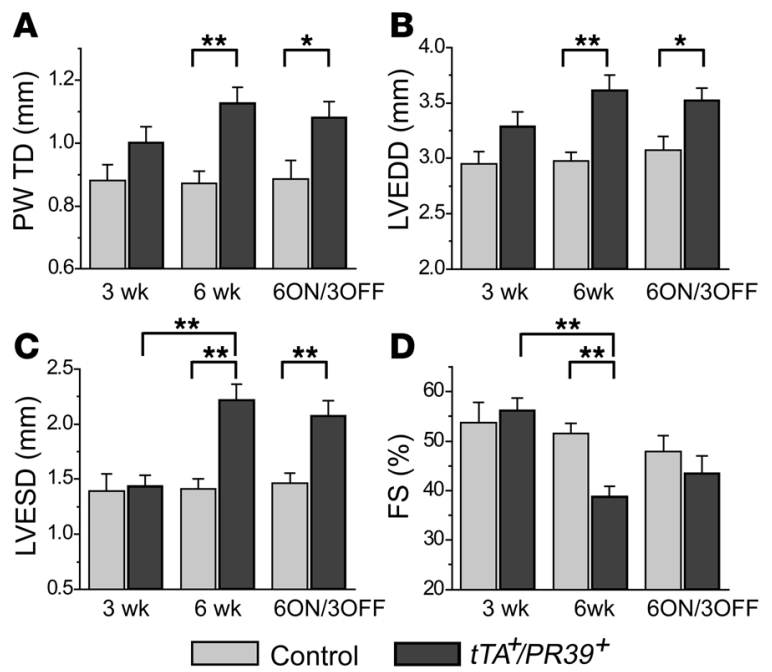

\section{Figure 5}

Echocardiographic analysis of myocardial function in $t T A^{+} / P R 39^{+}$mice. Echocardiographic analysis of LV function after 3 weeks or 6 weeks of PR39 induction or 6 weeks ON/3 weeks OFF (6PN/3OFF). (A) Posterior wall thickness in diastole (PWTD). (B) LV end-diastolic dimension (LVEDD). (C) LV end-systolic dimension (LVESD). (D) Fraction shortening (FS). Note the myocardial dilatation after 6 weeks' induction in tTA+/PR39+ mice maintained after PR39 gene suppression (6ON/3OFF mice). $3 \mathrm{wk}, n=10 \mathrm{mice} /$ group; $6 \mathrm{wk}, n=15 \mathrm{mice} / \mathrm{group} ; 6 \mathrm{ON} / 3 \mathrm{OFF}$, $n=7$ mice/group. ${ }^{*} P<0.05 ;{ }^{* \star} P<0.001$.

The hypothesis that increased endothelial cell mass induces NO production that in turn leads to myocardial hypertrophy (Figure 8) is further supported by our observations that direct exposure of cardiomyocytes to PR39 does not result in activation of a hypertrophic program or in an increase in protein synthesis that would be expected in a hypertrophic response. To further explore the functional significance of PR39-induced, angiogenesis-driven hypertrophy, we employed an acute MI model. We previously demonstrated that PR39 expression in the heart results in decreased infarct size, likely owing to increased vascular density of the myocardium (9), and that acute administration of PR39 reduces MI size, likely due to its ability to block I $\mathrm{B} \alpha \alpha$ degradation (11). Further examination of the model in this study demonstrated improved myocardial function in PR39 mice consistent with reduced infarct size. However, despite this improvement in cardiac performance, and hence, reduced $L V$ size and wall stress, we observed a significant increase in the size of myocytes in the unaffected free wall of the LV, consistent with a hypertrophic effect of PR39 expression.

To confirm that this effect of angiogenesis-driven hypertrophy is not PR39 specific, we employed VEGF-B, a growth factor with a known ability to induce cardiac angiogenesis. Intravenous administration of Ad-hVEGF-B 167 in the setting of acute MI resulted in increased angiogenesis in the unaffected free wall of the LV and improved cardiac performance, mimicking the effect of PR39. Furthermore, similar to PR39 mice, Ad-hVEGF-B ${ }_{167}$-treated mice also demonstrated increased myocyte size in the LV free wall. The ability of VEGF-B, an angiogenic growth factor completely unrelated to PR39, to induce myocardial hypertrophy further argues in support of the link between angiogenesis and hypertrophy.

The relationship between angiogenesis and hypertrophy has attracted considerable recent attention. In general, angiogenesis has been considered necessary to support ongoing hypertrophy induced by a hemodynamic stimulus such as pressure overload. Inhibition of angiogenic response has been demonstrated to lead to a rapid progression from compensated hypertrophy to heart failure $(7,20-22)$. In this study, we demonstrate that angiogenesis can induce myocardial hypertrophy even in the absence of a hemodynamic stimulus. This points out a complex role played by the heart's (and likely other organs') endothelium that involves not only participation in a stress response but also regulation of the organ's size. Interestingly, the increase in heart size observed in this study ceased once the vascular density returned to normal levels.

The concept of endothelial cell mass-driven regulation of organ size was suggested by Judah Folkman in the context of pro- and antiangiogenic treatment following organ injury (2). However, the ability of increased vascular mass to affect the size and function of a normal organ has not to our knowledge been previously demonstrated. This study demonstrates that an increase in the size of the vascular bed in the normal heart leads to increased cardiac mass and myocardial hypertrophy paralleled by increased cardiac performance.

\section{Methods}

\section{Generation of heart-specific inducible transgenic model $\left(t T^{+} / P R 39^{+}\right)$}

PR39 cDNA (GenBank no. NM_214450) was subcloned in bidirectional pBI-G Tet Vector (Clontech) sensitive to tTA. Flanked by 2 minimal CMV promoters, TRE controls LacZ as reporter gene and PR39 gene. In cell culture, inducible TRE-PR39/LacZ vector was expressed in Tet-Off MEF/3T3 cell line (Clontech) in the absence of doxycycline, and $\beta$-galactosidase activity was monitored by

Table 1

Pressure-volume loop analysis of cardiac function

\begin{tabular}{|c|c|c|c|c|c|c|}
\hline Parameter & Control 6 wk ON & $\begin{array}{l}t T A^{+} / P R 39^{+} \\
6 \text { wk ON }\end{array}$ & $\begin{array}{l}\text { Control } 6 \text { wk } \\
\text { ON + L-NAME }\end{array}$ & $\begin{array}{c}t T A^{+} / P^{2} 39^{+} \\
6 \text { wk ON + L-NAME }\end{array}$ & $\begin{array}{l}\text { Control } \\
60 N / 30 F F\end{array}$ & $\begin{array}{l}t T A^{+} / P R 39^{+} \\
60 \mathrm{~N} / 30 \mathrm{FF}\end{array}$ \\
\hline Cardiac output (ul/min) & $5,865 \pm 37$ & $2,918 \pm 107^{A}$ & $5,288 \pm 98$ & $4,239 \pm 110^{A, B}$ & $5,592 \pm 180$ & $9,046 \pm 239 A$ \\
\hline Ejection fraction (\%) & $59.61 \pm 0.31$ & $32.65 \pm 1.18^{A}$ & $52.46 \pm 0.62$ & $46.30 \pm 1.000^{A, B}$ & $66.07 \pm 1.44$ & $85.16 \pm 0.76^{A}$ \\
\hline Maximal power $(\mathrm{mmHg} / \mu \mathrm{l} / \mathrm{s})$ & $5.21 \pm 0.08$ & $3.05 \pm 0.16^{A}$ & $5.52 \pm 0.10$ & $4.44 \pm 0.14^{\mathrm{A}, \mathrm{B}}$ & $4.00 \pm 0.16$ & $8.71 \pm 0.27^{\mathrm{A}}$ \\
\hline$\tau(\mathrm{ms})$ & $7.00 \pm 0.09$ & $8.27 \pm 0.10^{A}$ & $5.50 \pm 0.07$ & $7.03 \pm 0.10^{\mathrm{A}, \mathrm{B}}$ & $7.79 \pm 0.18$ & $6.03 \pm 0.10^{A}$ \\
\hline $\mathrm{d} P / \mathrm{d} t \max (\mathrm{mmHg} / \mathrm{s})$ & $7,674 \pm 128$ & $4,468 \pm 101^{A}$ & $8,744 \pm 63$ & $7,020 \pm 129 \mathrm{~A}, \mathrm{~B}$ & $6,033 \pm 213$ & $8,809 \pm 167^{A}$ \\
\hline $\mathrm{d} P / \mathrm{d} t$ Min $(\mathrm{mmHg} / \mathrm{s})$ & $-8,042 \pm 140$ & $-4,368 \pm 93^{A}$ & $-8,331 \pm 61$ & $-6,328 \pm 138 \mathrm{~A}, \mathrm{~B}$ & $-5,129 \pm 174$ & $-7,503 \pm 123^{A}$ \\
\hline
\end{tabular}


A



B

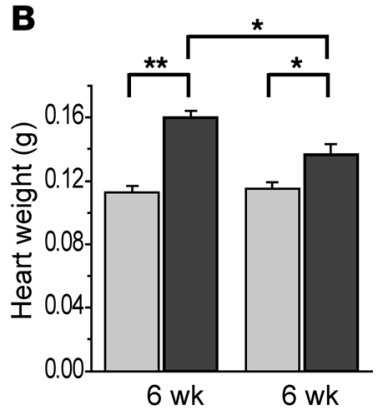

C

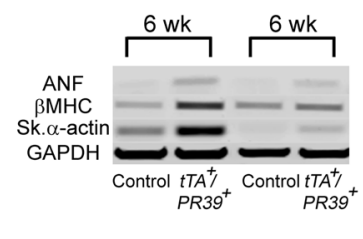

D

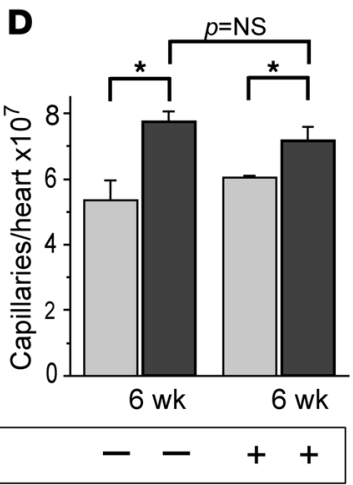

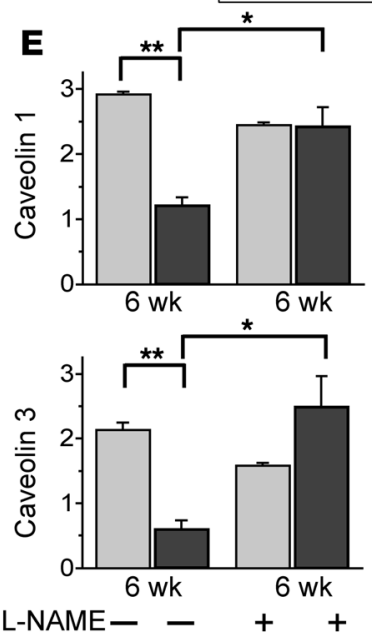

$\mathbf{F}$

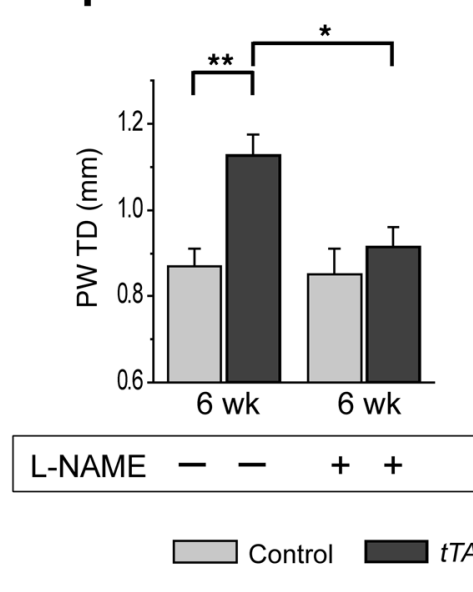

G

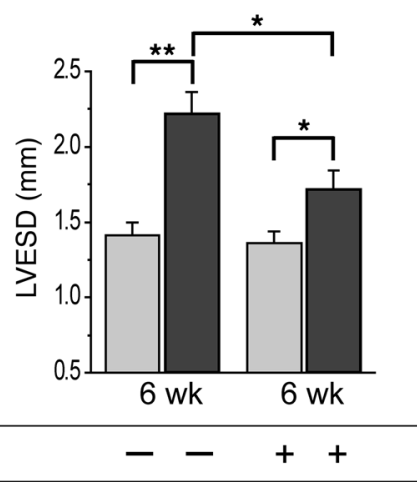

$T A^{+} / P R 39^{+}$

Figure 6

Cardiac hypertrophy reduction after L-NAME treatment. (A) Cycle GMP production in $t T A^{+} / P R 39^{+}$and control mouse hearts after 6 weeks of PR39 induction ( $n=4$ mice/group). (B) Heart weight measurements after 6 weeks of PR39 induction with and without L-NAME treatment ( $0.5 \mathrm{~g} / \mathrm{l}$ tap water). L-NAME was given during the last 3 weeks of a 6 -week induction period. Note reduction of the increase in heart size caused by PR39 induction (untreated, $n=16$ mice/group; L-NAME treated, $n=12$ mice/group). (C) RT-PCR analysis of hypertrophy marker expressions in L-NAME-treated versus untreated mice. GAPDH expression used as control. Representative data are shown. Note reduced expression of hypertrophic markers in L-NAME-treated mice. (D) Capillary mass of L-NAME-treated versus untreated mice. (E) Western blot analysis of expression of caveolin-1 (normalized to VE-cadherin) and caveolin-3 (normalized to skeletal $\alpha$-actinin) after 6 weeks of PR39 induction in the absence or presence of L-NAME ( $n=3$ mice/group). Note caveolin-1 and -3 expression restoration by L-NAME treatment. (F and G) Effect of L-NAME treatment on LV function. Echocardiographic assessment of posterior wall thickness in diastole in $\mathbf{F}$ and LV end-systolic dimension in G. Untreated, $n=15$ mice/group; L-NAME treated, $n=6$ mice/group. ${ }^{* \star} P<0.001 ;{ }^{*} P<0.05$.

a standard enzyme assay (Promega). As reference control, a non-inducible reporter $\beta$-galactosidase vector ( $\mathrm{PCMV} \beta$ ) was used. The TRE-PR39/LacZ construct was microinjected into fertilized mouse eggs. Founders were identified by PCR analysis, using specific primers for PR39: forward primer, $5^{\prime}$-CCCATCCATTCACTCACTGGA-3'; reverse primer, 5'-AAACCTCCCACACCTCCCC$3^{\prime}$. Transgenic mouse tTA/PR39 was obtained by crossing heart-specific driver line ( $\alpha$-MHC-tTA) (23) and responder line (TRE-PR39/LacZ). Double transgenic mice were genotyped by PCR with primers specific for $\mathrm{tTA}$ and PR39. Littermates inheriting only 1 transgene were used as controls. Transgene expression of PR39/LacZ was repressed by doxycycline in $\operatorname{diet}(200 \mathrm{mg} / \mathrm{kg}$ food) and induced on normal diet. Control mice were treated similarly. All animal experiments were approved by the Institutional Animal Care and Use Committees of Dartmouth College and University of Leuven.

\section{Transgene expression in mouse model}

PR39 transcript. The PR39 transcript was identified by RT-PCR analysis carried out using the PR39-specific primers shown above. GAPDH amplification was used as control (forward primer,
5'-CGTATTGGGCGCCTGGTCACCAGGGC-3'; reverse primer, 5'-GGCCATGAGGTCCACCACCCTGTTGC-3').

$\beta$-Galactosidase activity. $\beta$-Galactosidase activity in freshly harvested hearts or frozen sections was detected by staining with $\mathrm{X}-\mathrm{Gal}$ solution $[0.2 \% \mathrm{X}-\mathrm{Gal}$, $\left.2 \mathrm{mM} \mathrm{MgCl}_{2}, 5 \mathrm{mM} \mathrm{K}_{4} \mathrm{Fe}(\mathrm{CN})_{6} 3 \mathrm{H}_{2} \mathrm{O}, 5 \mathrm{mM} \mathrm{K}_{3} \mathrm{Fe}(\mathrm{CN})_{6}\right]$ (Promega). Frozen sections stained with $\mathrm{X}-\mathrm{Gal}$ solutions were counterstained with eosin.

\section{PECAM measurements}

Anti-PECAM Ab (Pharmingen) labeled with ${ }^{125}$ I (PerkinElmer Life Sciences) and mixed with nonspecific isotype control antibody labeled with ${ }^{131} \mathrm{I}$ was injected through a jugular vein catheter and allowed to circulate (24). After washing with bicarbonate-buffered saline, hearts were collected and weighed, and the radioactivity was counted on the $\gamma$-counter and reported per $1 \mathrm{~g}$ tissue.

\section{Histological assessments}

Serial $5-\mu \mathrm{m}$ heart sections (fixed in $4 \%$ paraformaldehyde and embedded in paraffin) were stained with $\mathrm{H} \& \mathrm{E}$ for overall morphology assessment, 
A

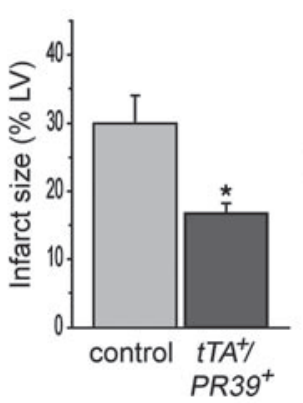

E

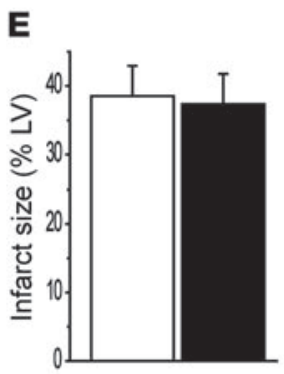

B
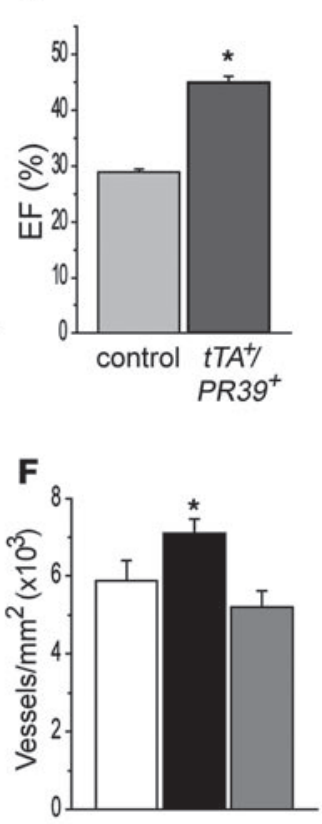

C
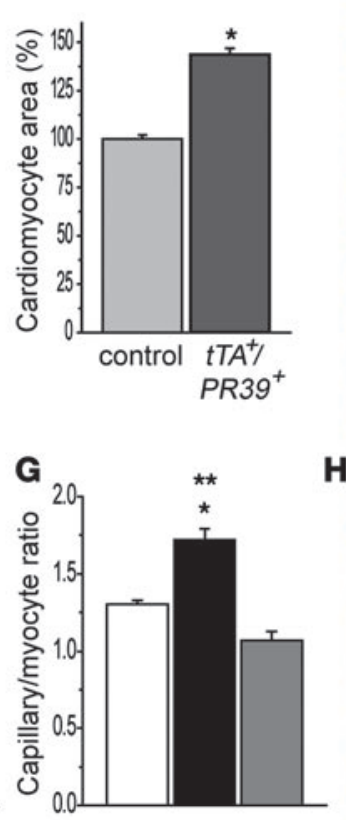

J

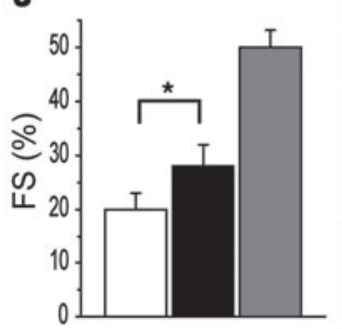

D

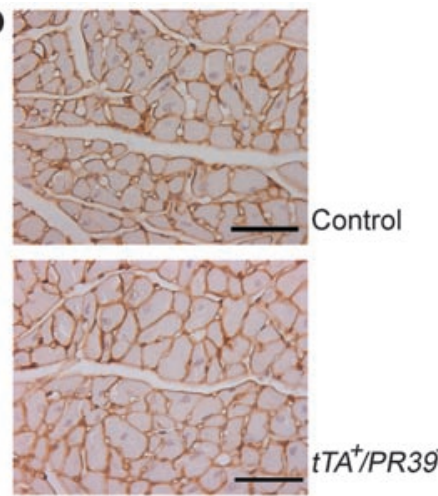

H

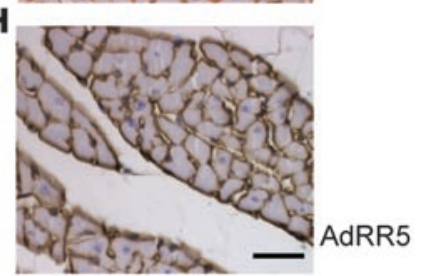

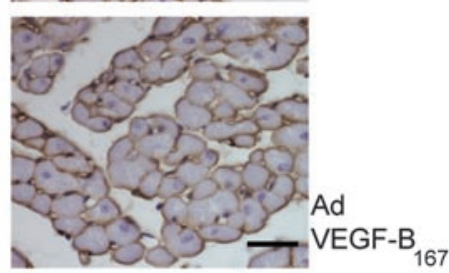

Sham

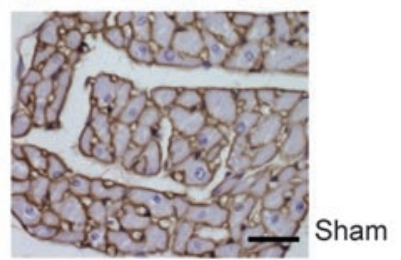

\section{Figure 7}

Angiogenesis-induced hypertrophy in the setting of MI. (A) PR39 induction in $t T A^{+} / P R 39^{+}$mice at the time of LAD ligation resulted in reduction of the infarct size 28 days after MI ( $n=6$ mice/group). (B) Ejection fraction (EF) assessed by pressure-volume loop analysis at 28 days after MI in induced $t T A^{+} / P R 39^{+}$and control mice. (C and D) Cross-sectional area of cardiomyocytes in the free (normal) LV wall myocardium in induced $t T A^{+} / P R 39^{+}$and control mice expressed as percentage of control. (D) Representative laminin immunostaining of LV myocardium from the 2 mouse groups. (E) Infarct size 28 days after MI in C57BL/6 mice exposed to Ad-hVEGF-B 167 (black bars) or control virus (Ad-RR5, white bars). Sham, gray bars. Ad-hVEGF-B ${ }_{167}, n=16$; Ad-RR5, $n=12$; sham, $n=5$. (F and $\left.\mathbf{G}\right)$ Capillary count $(\mathbf{F})$ and capillary/myocyte ratio $(\mathbf{G})$ in mice exposed to Ad-VEGF-B 167 or Ad-RR5 28 days after Ml or in sham-operated mice. (H and I) Cardiomyocyte hypertrophy in the remote myocardium. Laminin staining $(\mathbf{H})$ and quantitative analysis (I) of cardiomyocyte area in the normal myocardium of C57BL/6 mice 28 days after Ml or a sham procedure and exposed to Ad-hVEGF-B ${ }_{167}$ or Ad-RR5. (J) LV function (fractional shortening) 28 days after MI or a sham procedure in C57BL/6 mice exposed to Ad-hVEGF-B 167 or Ad-RR5. ${ }^{*} P<0.05 ;{ }^{* *} P<0.05$ versus sham. Scale bars: $50 \mu \mathrm{m}$.

Masson's trichrome for detection of fibrosis, and biotinylated isolectin B4 (Vector Laboratories) for capillary counting. Capillary density was quantified on serial $2,500 \mu \mathrm{m}^{2} / 5 \mu \mathrm{m}$ sections. Frozen sections (embedded in OCT compound) were used for detection and quantification of apoptosis based on TUNEL technology (Roche Diagnostics). Proliferating nuclei were labeled with Ki67 Ab (Dako), and nuclear staining was done using DAPI (Invitrogen).

\section{Cyclic GMP assay}

Quantification of cGMP directly from heart tissue homogenates were performed by a competitive EIA assay (Cayman Chemical). Frozen tissues were homogenized in ice-cold $6 \%$ TCA in the presence of $300 \mu \mathrm{M}$ PDE inhibitor 3-isobutyl-1-methylxanthine (IBMX). After centrifugation, TCA was extracted by washing supernatants with water-saturated diethyl ether. The remaining aqueous extract was dried under nitrogen. Cyclic GMP was determined in acetylated samples and normalized to tissue weight.

\section{Cardiomyocyte isolation and culture experiments}

Adult mouse cardiomyocyte isolation. Adult mouse cardiomyocyte isolation was performed using the Langendorff apparatus (Cellutron Life Technologies). After digestion, isolated cardiomyocytes were plated on laminin. Three hours later, cell size was measured in captured images with a Nikon inverted microscope, using ImageJ software.

Neonatal rat cardiomyocytes. Neonatal rat cardiomyocytes were isolated from hearts of 3-day-old Harlan Sprague-Dawley rats using the Neonatal Cardiomyocyte Isolation System (Worthington Biochemical Corp.). 




Figure 8

Angiogenesis-induced hypertrophy. A proposed sequence of events resulting in hypertrophy induction. Increased angiogenic growth factor presence in the tissue stimulates endothelial cell proliferation (step 1), which in turn leads to increased vascular density (step 2) and higher baseline NO production (step 3). NO induction of cellular hypertrophy (step 4) results in eventual normalization of vascular density and reduction in angiogenic growth factor concentration (step 5), terminating the process.

To selectively enrich for myocytes, dissociated cells were preplated for 1 hour. The resulting suspension of cardiomyocytes was plated (density, 125,000 cells $/ \mathrm{cm}^{2}$ ) on fibronectin and cultured for 24 hours in DMEM supplemented with $10 \% \mathrm{FBS}$ and $100 \mu \mathrm{M}$ BrdU. All experiments were done in the serum-free conditions 24 hours after changing to the serumfree medium (DMEM supplemented with $1 \mathrm{mg} / \mathrm{ml} \mathrm{BSA}, 10 \mu \mathrm{g} / \mathrm{ml}$ transferrin, and $10 \mu \mathrm{g} / \mathrm{ml}$ insulin).

Adenoviral transduction. Myocytes were exposed overnight to Ad-PR39 or Ad-GFP at $100 \mathrm{MOI}$ in serum-free medium. The medium was then replaced with fresh serum-free medium, and cells were used for the experiments described below. Under these conditions, approximately $90 \%$ of cells were infected based upon GFP expression as determined by fluorescence microscopy for Ad-GFP-treated cells (data not shown).

PR39 and angiotensin II peptide treatment. Freshly isolated cardiomyocytes were incubated with synthetic peptide PR39 $(1 \mu \mathrm{M})$ (Polypeptide Laboratories) or human synthetic angiotensin II (1 $\mu \mathrm{M})$ (Sigma-Aldrich) in serum-free medium.

${ }^{3} \mathrm{H}$-leucine incorporation. Ad-PR39-transduced or PR39- or angiotensin IItreated serum-starved ( 36 hours) cardiomyocytes were exposed to $2 \mu \mathrm{Ci} / \mathrm{ml}$ ${ }^{3} \mathrm{H}$-leucine (PerkinElmer Life Sciences) 12 hours before harvest. The total radioactivity of ${ }^{3} \mathrm{H}$-leucine incorporated into protein was determined in TCA-precipitated protein solubilized in $0.5 \mathrm{M} \mathrm{NaOH}, 0.1 \%$ Triton $\mathrm{X}-100$. ${ }^{3} \mathrm{H}$-Leucine incorporation was reported relative to total protein as determined by BCA assay (Pierce Biotechnology).

Cell size measurements in culture. After 72 hours, cardiomyocytes cultured in serum-free medium, transduced with Ad-PR39, or treated with PR39 or angiotensin II cells were fixed in 4\% PFA and permeabilized with 2\% PFA, $0.1 \%$ Triton X-100, 0.1\% NP-40. Morphological and morphometric analyses were carried out by immunostaining with anti $\alpha$-actinin sarcomeric $\mathrm{Ab}$ 1/50 (Sigma-Aldrich) and visualizing with an Olympus IX-71 microscope. Myocyte area was measured in captured images using ImageJ software.

\section{Hypertrophic markers}

Analysis of ANF, $\beta$-MHC, and skeletal $\alpha$-actin gene expression was performed by RT-PCR. Total RNA was isolated from LV with Tri Reagent (Molecular Research Center Inc.) and reverse-transcribed with Thermo-
Script RT-PCR System (Invitrogen). PCR reaction was carried out using specific primers (courtesy of C. Patterson, University of North Carolina, Chapel Hill, North Carolina, USA). GAPDH expression was used as control.

\section{Western blots}

Immunoblotting. Proteins and LV tissue homogenates were extracted in RIPA lysis buffer supplemented with protease inhibitor cocktail (Roche). The lysates were clarified by ultracentrifugation $\left(12,000 \mathrm{~g}, 15\right.$ minutes at $\left.4^{\circ} \mathrm{C}\right)$, and $50 \mu \mathrm{g}$ protein extract, determined by BCA assay, was subjected to SDS-PAGE. Following transfer to Immobilon membranes (Millipore), the membranes were blocked with $5 \%$ nonfat dried milk in TBS, $\mathrm{pH} 7.4$, containing $0.1 \%$ Tween-20, and immunoblotted with anti-phospho-Akt (Ser473) (Cell Signaling), anti-Akt (Cell Signaling), anti-phosho-p44/42 MAPK (Erk1/2; Cell Signaling), anti-p44/42 MAPK (Erk1/2; Cell Signaling), anti-GATA4 antibody (Santa Cruz Biotechnology Inc.), anti-VE-cadherin (Santa Cruz Biotechnology Inc.), anti-caveolin-1 (BD - Pharmingen), anti-caveolin-3 (BD - Transduction Laboratories), anti- $\alpha$-actinin sarcomeric (Sigma-Aldrich), anti-GAPDH (Research Diagnostics Inc.), anti-HIF-1 $\alpha$ (Novus Biologicals), anti-endothelial NOS3, and inducible NOS2 (Santa Cruz Biotechnology Inc.). Immunoreactive bands were visualized using the horseradish peroxidase-conjugated secondary antibody and enhanced chemiluminescence substrate (Pierce Biotechnology).

\section{MI model}

$t T A^{+} / P R 39^{+}$mice. Control and $t T A^{+} / P R 39^{+}$mice (3-4 months old) were anesthetized and intubated. Using aseptic technique, the chest was opened between the third and fourth ribs to expose the heart. A 8-0 prolene suture was then used to ligate the LAD coronary artery. EKG S-T segment monitoring and visual observation of blanching were used to confirm a complete ligation. The chest was then closed and mice were recovered. At the time of LAD ligation, doxycycline food was replaced with regular food to activate the PR39 gene expression. 28 days later mice underwent echocardiography followed by pressure-volume loop study of cardiac function.

$A d-b V E G F-B_{167}$ treatment. C57BL/6 mice (3-4 months old) underwent induction of MI by ligation of LAD coronary artery as described above. Sham operation included all procedures except ligation of the LAD. Immediately after LAD ligation, $3 \times 10^{9} \mathrm{pfu}$ Ad-hVEGF-B 167 or control adenovirus Ad-RR5 expressing no transgene was intravenously injected into the tail vein. VEGF-B plasma level was measured using ELISA (R\&D Systems). Adenoviruses were constructed by cloning the human VEGF-B 167 cDNA into the pACCMVpLpA plasmid, as previously described (25).

Histology in remote myocardium. At 28 days after MI, hearts were harvested for histological analysis as previously described $(25,26)$. Briefly, mice were anesthetized and perfused via the abdominal aorta with a saline solution followed by $1 \%$ phosphate-buffered paraformaldehyde, and fixed hearts were cryoembedded or embedded in paraffin. Serial sections were immunostained with the rat anti-CD31 Ab (Becton Dickinson) for vessel counting, anti-laminin $\mathrm{Ab}$ (Sigma-Aldrich) for myocyte area measurements, and double stained with CD31 and laminin for capillary/cardiomyocyte ratio analysis. Morphometric analyses were performed using KS300 image analysis and ImageJ software.

\section{Echocardiography and hemodynamic measurements}

Echocardiography. Mice were sedated with a mixture of ketamine/acepromazine (1.8 mg/0.04 mg per mouse). Echo images were acquired in M-mode standard procedure.

Cardiac pressure-volume loop analysis. A 1.4-F pressure-volume catheter was introduced into the LV via the right carotid artery to determine pressurevolume loop variables. Animals were ventilated and anesthetized with $2 \%$ isoflurane, and body temperature was maintained by standard methods. 
Baseline parameters were recorded 30 minutes after stabilization. Parallel conductance was assessed with hypertonic saline. Raw data were imported in PVAN 3.2 (Millar) for the assessment of cardiac performance parameters and parallel conductance.

\section{Statistics}

Data are presented as mean \pm SEM. Differences between groups were considered statistically significant if $P<0.05$ as determined by a Student $t$ test, two-tailed distribution. Differences in hemodynamic parameters resolved by echocardiography and pressure-volume loops were considered significant at $P<0.05$ by ANOVA Dunnett's test and mixed-mode analyses (20-30 loops/mouse, 5 mice/group).

\section{Acknowledgments}

This work was supported in part by NIH grant HL53793 (M. Simons). We would like to thank Steven Fiering for TRE-PR39/
LacZ mouse generation, Ebo DeMuinck for advice with echo and pressure-volume loop studies, and Samantha Shipman for technical assistance with mouse breeding and genotyping (Dartmouth Medical School). This work was supported in part by a grant from Philip Morris (to L. Moons).

Received for publication March 5, 2007, and accepted in revised form August 29, 2007.

Address correspondence to: Michael Simons, Section of Cardiology, Dartmouth-Hitchcock Medical Center, One Medical Center Drive, Lebanon, New Hampshire 03756, USA. Phone: (603) 650-3540; Fax: (603)650-5171; E-mail: michael.simons@dartmouth.edu.

Daniela Tirziu and Emmanuel Chorianopoulos contributed equally to this work.
1. Dallabrida, S.M., et al. 2003. Adipose tissue growth and regression are regulated by angiopoietin-1. Biochem. Biophys. Res. Commun. 311:563-571.

2. Folkman, J. 1998. Is tissue mass regulated by vascular endothelial cells? Prostate as the first evidence. Endocrinology. 139:441-442.

3. Greene, A.K., et al. 2003. Endothelial-directed hepatic regeneration after partial hepatectomy. Ann. Surg. 237:530-535.

4. Hunter, J.J., and Chien, K.R. 1999. Signaling pathways for cardiac hypertrophy and failure. N. Engl. J. Med. 341:1276-1283.

5. Lorell, B.H., and Carabello, B.A. 2000. Left ventricular hypertrophy: pathogenesis, detection, and prognosis. Circulation. 102:470-479.

6. Gavin, J.B., Maxwell, L., and Edgar, S.G. 1998 Microvascular involvement in cardiac pathology. J. Mol. Cell. Cardiol. 30:2531-2540.

7. Shiojima, I., et al. 2005. Disruption of coordinated cardiac hypertrophy and angiogenesis contributes to the transition to heart failure. J. Clin. Invest. 115:2108-2118. doi:10.1172/JCI24682.

8. Suzuki, G., Lee, T.C., Fallavollita, J.A., and Canty, J.M., Jr. 2005. Adenoviral gene transfer of FGF-5 to hibernating myocardium improves function and stimulates myocytes to hypertrophy and reenter the cell cycle. Circ. Res. 96:767-775.

9. Li, J., et al. 2000. PR39, a peptide regulator of angiogenesis. Nat. Med. 6:49-55.

10. Hu, R.G., et al. 2005. The N-end rule pathway as a nitric oxide sensor controlling the levels of mul- tiple regulators. Nature. 437:981-986.

11. Bao, J., et al. 2001. PR-39 and PR-11 peptides inhibit ischemia-reperfusion injury by blocking proteasome-mediated I kappa B alpha degradation. Am.J. Physiol. Heart Circ. Physiol. 281:H2612-H2618.

12. Bellomo, D., et al. 2000. Mice lacking the vascular endothelial growth factor-B gene (Vegfb) have smaller hearts, dysfunctional coronary vasculature, and impaired recovery from cardiac ischemia. Circ. Res. 86:E29-E35.

13. Tomanek, R.J., and Busch, T.L. 1998. Coordinated capillary and myocardial growth in response to thyroxine treatment. Anat. Rec. 251:44-49.

14. Dor, Y., et al. 2002. Conditional switching of VEGF provides new insights into adult neovascularization and pro-angiogenic therapy. EMBO J. 21:1939-1947.

15. Drab, M., et al. 2001. Loss of caveolae, vascular dysfunction, and pulmonary defects in caveolin-1 gene-disrupted mice. Science. 293:2449-2452.

16. Razani, B., et al. 2001. Caveolin-1 null mice are viable but show evidence of hyperproliferative and vascular abnormalities. J. Biol. Chem. 276:38121-38138.

17. Woodman, S.E., et al. 2002. Caveolin-3 knock-out mice develop a progressive cardiomyopathy and show hyperactivation of the $\mathrm{p} 42 / 44$ MAPK cascade. J. Biol. Chem. 277:38988-38997.

18. Brunner, F., Andrew, P., Wolkart, G., Zechner, R., and Mayer, B. 2001. Myocardial contractile function and heart rate in mice with myocyte-specific overexpression of endothelial nitric oxide synthase.
Circulation. 104:3097-3102.

19. Brunner, F., et al. 2003. Attenuation of myocardial ischemia/reperfusion injury in mice with myocytespecific overexpression of endothelial nitric oxide synthase. Cardiovasc. Res. 57:55-62.

20. Izumiya, Y., et al. 2006. Vascular endothelial growth factor blockade promotes the transition from compensatory cardiac hypertrophy to failure in response to pressure overload. Hypertension. 47:887-893.

21. Shiojima, I., and Walsh, K. 2006. Regulation of cardiac growth and coronary angiogenesis by the Akt/ PKB signaling pathway. Genes Dev. 20:3347-3365.

22. Sano, M., et al. 2007. p53-induced inhibition of Hif- 1 causes cardiac dysfunction during pressure overload. Nature. 446:444-448.

23. Yu, Z., Redfern, C.S., and Fishman, G.I. 1996. Conditional transgene expression in the heart. Circ. Res. 79:691-697.

24. Eppihimer, M.J., et al. 1998. Differential expression of platelet-endothelial cell adhesion molecule-1 (PECAM-1) in murine tissues. Microcirculation. 5:179-188.

25. Heymans, S., et al. 1999. Inhibition of plasminogen activators or matrix metalloproteinases prevents cardiac rupture but impairs therapeutic angiogenesis and causes cardiac failure. Nat. Med. 5:1135-1142.

26. Luttun, A., et al. 2002. Revascularization of ischemic tissues by PlGF treatment, and inhibition of tumor angiogenesis, arthritis and atherosclerosis by anti-Flt1. Nat. Med. 8:831-840. 